\title{
Le Règlement des Différends entre les Etats Africains
}

\author{
Par Maria Magdalena Kenig
}

Le règlement des différends internationaux en Afrique est un des problèmes-clés des relations inter-africaines. L'analyse de ce processus et l'ẻvaluation de son efficacité sont conditionnées, à mon avis, par deux éléments essentiels, et notamment les dispositions en la matière contenues dans les traités, et la pratique internationale. De même, au point de vue de la conception de la formation du droit international africain, ces deux éléments sont essentiels à égalité.

\section{Modes de règlement des différends internationaux - cas puisés dans le droit positif af ricain}

La revue des accords bi- et multilatéraux conclus entre les Etats africains nous permet d'apercevoir et de déterminer les tendances que l'on observe en Afrique en ce qui concerne les modes de règlement des différends. Une importance particulière revient ici à l'analyse des accords multilatéraux-, ceux-ci du fait de leur caractère influent considérablement sur la formation de normes du droit international soit à l'échelle d'une région ou sous-région, soit à celle d'un continent, comme c'est le cas de la Charte de l'Organisation de l'Unité Africaine. Celle-ci stipule que les Etats africains régleront tous les différends par voie de négociation, de médiation, de conciliation ou d'arbitrage. ${ }^{1}$

\subsection{Modes diplomatiques - négociations directes}

A l'époque contemporaine, les négociations sont en train d'être institutionnalisées dans les actes fondamentaux du droit international. On rappellera que la Charte de l'Organisation de l'Unité Africaine était, à côté de la Charte des Nations Unies et de la Charte de l'Organisation des Etats Americains (OAS) l'un des premiers documents dans l'histoire du droit international positif consacrant voire institutionnalisant les négociations en tant que mode indépendant de régler les différends internationaux. Parmi les modes de règlement des litiges, la première place revient aux négociations, le plus souvent citées

1 Les dispositions contenues dans la Charte de l'OUA, et celles du Protocole de Médiation, de Conciliation et d'Arbitrage concernant le règlement des différends ont fait objet d'un nombre important d'analyses juridiques dues, entr'autres, à M. Bedjaoui, J. M. Bipoun-Woum, F. Berella, B. Boutres-Ghah, Zd. Cervenka, Ph. Chapal, J. Diaite, T. O. Elias, P. F. Gonidec, M. M. Kenig, K. Morjane, J. P. Quenedec, R. A. Touzmoukhamiedov, R. Yakemtchouk, K. Hutschenreuter. 
dans les accords conclus entre les Etats africains. Il est caractéristique que les procédures y sont généralement mentionnées dans l'ordre conforme à l'art. 33 de la Charte de l'ONU. De nombreux cas semblent confirmer le fait que les Etats africains ont reconnu la nécessité de recourir à des négociations plutôt que de voir les différends soumis à la compétence juridique. Des dispositions circonstanciées des accords contiennent des réserves explicites ou implicites qui excluent certains modes de règlement des différends, sans, cependant, exclure les négociations directes. D'ailleurs, même dans le cas pareil, une telle exclusion demeurerait inefficace, car les négociations restent toujours cet élément fondamental par lequel un différend se manifeste. Même dans le cas où un accord donné ne mentionne pas les négociations, il convient de supposer qu'un recours à tout autre mode de règlement ne serait possible qu'après l'épuisement des négociations directes. Au contraire, est supposée exclue toute autre solution que les Parties contractantes n'avaient pas citée parmi les modes de règlement des différends prévus par le traité.

Les accords conclus entre les Etats africains utilisent diverses définitions pour désigner la procédure des négociations. Ainsi, p.ex., le Traité du Comité Inter-Etats pour l'Aménagement du fleuve Sénégal (Dakar, le 16 février 1964) évoque une entente directe entre les Etats (art. 15). ${ }^{2}$ Nombreux sont les traités où le recours à d'autres procédures est nécessairement précédé par les tentatives soit de négociations directes soit de "règlement à l'amiable du différend". Il en est de même de l'art. 56 du traité instituant la Communauté Economique des Etats de l'Afrique de l'Ouest (C.E.D.E.A.O.-Lagos, le 28 mai 1975) ledit article imposant la nécessité de règlement à l'amiable des différends avant que ceux-ci ne soient portés devant le Tribunal de la Communauté. ${ }^{3}$ Rappellons également la Convention relative à la Navigation et à la Coopération Economique entre les États du bassin du Niger (Niamey, du 24 au 26 octobre 1963) où la nécessité de régler les différends à l'amiable est citée à la première place, précédant d'autres modes de règlement des litiges. ${ }^{4}$

Il est intéressant que le droit international positif africain a adopté les consultations en tant que forme indépendante des négociations directes en vue du règlement d'un différend. ${ }^{5}$ Ainsi, p.ex., l'art. 17 du traité relatif au transports aériens en Afrique (Yaoundé, le 28 mars 1961) stipule que les différends entre les Etats contractants seront réglés par voie de consultation ou, à défaut, seront soumis à l'arbitrage. ${ }^{6}$ C'est le genre de consultations que l'on peut définir comme une sorte de pourparlers directs entre les Parties à un litige. Des consultations à caractère mixte sont mentionnées dans l'art. 4 du Pacte de

2 A.F.D.I. 1971 , p. 737.

3 Af rique Contemporaine, 1976, no 88, p. 20.

4 Droit International et Science Politique, Recueil de Textes, Paris 1967, p. 321.

5 En 1978, à la conférence de Montreux consacrée au règlement: des différends internationaux, la Pologne, soutenue-par d'autres pays socialistes, a presenté un document proposant de mettre les consultations en tant que moyen indépendant sur la liste de modes de règlement des différends. L'art. 41 de la Convention de Vienne relative à la succession des Etats par rapport aus traités prévoit les consultations en tant que mode indépendant du règlement des litiges.

6 Revue Française de Droit Aérien, 1963, p. 333. 
Défense de l'Union Africaine et Malgache (Tananarive, le 9 septembre 1961) aux termes duquel les Parties conviennent de "se consulter quant aux moyens à adopter dans chaque cas où de l'avis de l'une d'elles, l'intégrité territoriale, l'indépendance politique ou la sécurité de l'une des Parties serait menacée".?

On soulignera que des traités bilatéraux mentionnent souvent les négociations comme moyen unique de régler les différends. Ainsi, p.ex., les différends relatifs à l'application du traité frontalier conclu entre la République Islamique de Mauritanie et la République du Mali seront réglés par voie diplomatique. ${ }^{8}$ Il en est de même de l'accord sur les frontières algéro-marocain relatif à l'armistice qui stipule que tout différend survenant entre les Etats doit être réglé par voie des négociations. ${ }^{9}$

\section{Négociations indirectes}

Les négociations indirectes, d'est-à-dire les négociations menées avec la participation d'un élément tiers, ont également leur place parmi les procédures citées par le droit international positif africain. Le recours aux bons offices d'une tierce Puissance, d'une personnalité qualifiée ou d'un organisme international a lieu dans le cas où les Parties ne peuvent pas régler le différend qui les oppose par voie des négociations directes. En principe, les négociations indirectes ne sont qu'un instrument auxiliaire utilisé en vue de régler le différend. Les formes traditionnelles des négociations indirectes telles que les mentionnent les conventions de la Haye, et notamment bons offices, médiations, conciliation et enquête ont subi, en pratique, des transformations et modifications diverses; ainsi, il est courant que l'on institue des organismes qualifiés réunissant les éléments principaux des modes jusqu'alors traités séparément, dont le but consistait à régler les litiges de façon indirecte.

La Charte de l'OUA et le Protocole de Médiation, de Conciliation et d'Arbitrage mentionnent, en principe, deux modes des négociations indirectes, à savoir la médiation et la conciliation, bien que le Protocole fasse également état d'une sorte de procédure d'enquête. ${ }^{10}$ Ainsi, l'art. 18 dudit Protocole stipule que dans la situation où il aura été nécessaire de recourir à la procédure d'enquête afin d'établir la matérialité des faits relatifs à l'objet du litige, les Parties au différend de même que tous les Etats-membres assureront leur concours en la matière. La disposition ci-dessus exprime une tendance à reconnaître l'enquête comme une partie intégrante soit de la procédure de conciliation ou d'arbitrage soit de la procédure judiciaire.

Il semble également que la procédure de médiation présentée dans l'art. 21 du Protocole contient les éléments propres aussi bien aux bons offices qu'à la médiation elle-même. Le

\footnotetext{
Polityka zagranic zna państw afrykańskich/Politique extérieure des Etats africains/, Warszawa 1970, p. 155.

Af rique Contemporaine, 1963, no 9, p. 6.

Zbiór Documentów/Recueil de Documents/, 1963, no 9-10, pp. 1465-1467.

10 Organizacija Afrikanskoge Jediastva, Moskva 1965, pp. 35 et suiv., 115 et suiv.
} 
médiateur propose des solutions du différend qui, une fois acceptées par les Parties, deviennent une base obligatoire d'un accord.

Les dispositions relatives aux négociations indirectes considérées comme mode de règlement des différends sont contenues dans un bon nombre de traités bi- et et multilatéraux réglant ces procédures de façon plus ou moins précise et instituant, par ailleurs, des organismes permanents de médiation, de conciliation, des commissions de bons offices ou, éventuellement, des commissions ad hoc. Récemment, on observe un vif intérêt porté par des Etats, principalement du Tiers monde, aux négociations indirectes dont, en premier lieu, à la conciliation; ceci est dû au fait que les organismes de conciliation n'étant pas tenus d'appliquer les règles juridiques conviennent mieux à des Etats nouveaux dont l'attitude à l'égard des normes du droit international universel est plutôt méfiante.

Ces tendances se réflètent également dans le droit international positif africain où les négociations indirectes, et surtout la conciliation sont souvent citées comme le principal sinon l'unique mode de règlement des différends. Ainsi, p.ex., la Charte de l'Organisation Inter-Africaine et Malgache (Lagos, le 20 décembre 1962) stipule, au chapitre VIII, que "les Hautes Parties contractantes s'engagent à régler tous leurs différends par des moyens pacifiques et, à cette fin, décident de conclure un traité séparé instituant une commission permanente de conciliation ". ${ }^{11}$ Un autre exemple d'un traité international qui prévoit la conciliation et la médiation comme modes de règlement des différends est le traité instituant le Comité Inter-Etats pour l'Aménagement du fleuve Sénégal. Un grand nombre d'accords internationaux ne font pas de distinction entre les modes de négociations indirectes; ces accords statuent de façon générale sur le règlement des différends à l'intermédiaire p.ex., d'organismes internationaux, il y est question des négociations ou bien de voie diplomatique ce qui veut dire que toutes les procédures diplomatiques peuvent être utilisées. ${ }^{12}$

De nombreux traités internationaux conclus entre les Etats africains, surtout les traités instituant les organisations internationales, attribuent des compétences en matière de règlement des différends à certains des organes desdites organisations, le plus souvent supérieurs, composés généralement de représentants de tous les Etats-membres. Il semble bien que c'est l'une de ces formes de négociations que l'on peut définir comme "négociations collectives", celles-ci, à leur tour, ayant pris forme de ladite diplomatie de conférence qu'il ne faut pas confondre avec les négociations multilatérales. Dans le cas des négociations collectives citées plus haut, les problèmes litigieux sont en principe communs à tous les Etats-membres de l'organisation, surtout lorsqu'ils restent en rapport avec l'interprétation et l'application du traité. Ainsi leur solution à l'intérieur du groupe est l'une des formes les plus pratiques des négociations. Car il ne faut pas oublier

11 Documentation française, 1965, no 3.159, Notes et Etudes Documentaires, p. 40.

12 Cf. p. ex., la Convention relative à la navigation et à la cooperation économique entre les Etats du bassin du Niger, Niamey, le 26 octobre 1963, citée plus haut. 
que le mode de règlement d'un différend proposé par l'organe suprême de l'organisation a été mis au point pendant la durée des négociations le concernant.

Ainsi, p.ex., la première institution appelée à régler les différends relatifs à l'interprétation et à l'application du traité sur la Communauté de l'Afrique de l'Est (Arusha, le ler décembre 1967) est le Conseil du Marché Commun et ce n'est qu'après l'échec de ses tentatives, ou dans le cas où les Parties auront rejeté les solutions proposées que le litige pourra être porté devant le Tribunal du Marché Commun. ${ }^{13}$ Il en est de même de la Conférence des Chefs d'Etat et de Gouvernement près l'Organisation de l'Unité Africaine qui, en tant qu'organe suprême de l'OUA se réserve le droit de décision dans toutes les affaires relatives à l'interprétation et à l'application de la Charte. Rappellons également la Convention et le statut relatifs à la mise en valeur du bassin du Tchad (Fort Lamy, le 22 mai 1964) qui prévoit que tout différend sera soumis à la Commission de Médiation, de Conciliation et d'Arbitrage de l'OUA. ${ }^{14}$

Les institutions des organisations internationales participant au processus de règlement des différends n'ont à leur disposition que des résolutions, et ne tranchent, en principe, pas les litiges. Leur contribution consiste plutôt à assister les Parties au conflit, et à rechercher, sur la base de l'organisation elle-même, des solutions qui seraient acceptables par lesdites Parties. Quelles que soient les procédures, toutes sont de caractère indirect.

Nous aimerions évoquer ici une disposition contenue dans le statut du Fonds Africain de Développement (Abidjan, le 29 novembre 1972) aux termes de laquelle tous les problèmes relatifs à l'interprétation et à l'application du traité "sont soumis pour décision au Conseil d'administration (...) mais tout participant peut demander que la question soit portée devant le Conseil des Gouverneurs (organe suprême - N. de l'A.) dont la décision est sans appel. ${ }^{15}$

Pareillement, une décision contenue dans l'art. 9 § 3 du Statut de l'Union Economique et Douanière de l'Afrique Centrale institue le Conseil (organe suprême) - l'unique institution qualifiée à "arbitrer les différends qui peuvent surgir entre les Etats-membres ". ${ }^{16} \mathrm{On}$ voit ainsi que certains traités attribuent aux institutions des organisations internationales les compétences judiciaires comprises au sens large du mot. Ces institutions donc contribuent à un rapprochement entre le mode diplomatique et le règlement judiciaire.

\subsection{Règlement judiciaire}

La plupart des traités multilatéraux conclus entre les Etats africains prévoient également le règlement judiciaire des différends. Cette catégorie englobe la juridiction internationale au sens propre du mot, et l'arbitrage international. Par le terme "juridiction inter-

13 The Basic Documents of African Affairs, Oxford 1971, p. 88 et suiv.

14 Revue juridique et politique, 1965, no 2, p. 307.

15 Journal Officiel de la République Française. 12 II 1978, p. 704.

16 Af rique Contemporaine, 1966, no 18. 
nationale" on comprend parfois tout mode pacifique de règlement des différends qui consiste à soumettre un différend à un élément tiers, en vue de son règlement, sur la base des règles juridiques en vigueur, et de façon qui serait obligatoire pour les Parties. Le terme "juridiction" au sens propre du mot s'étend uniquement à des cours internationales permanentes dotées d'un statut définissant leurs fonctionnements respectifs et les règles juridiques qui sont le fondement de la jurisprudence.

Indépendemment des conséquences qui découlent de la distinction entre ces deux procédures de règlement judiciaire de différends (l'arbitrage suppose théoriquement une participation plus large des Etats-Parties au procédé juridique tandis que la cour internationale est censée garantir plus d'objectivité dans les jugements), il existe dans la doctrine une concordance d'opinions appuyée par la pratique comme quoi l'attitude des Etats à l'égard des modes judiciaires de règlement des différends est pleine de réserve. Les Etats craignent un risque lié à la nécessité de se conformer à la sentence qui aurait pu leur être défavorable tandis que l'avenir pourrait leur apporter une solution plus à leur gré.

En dépit de ces craintes, la plupart des traités multilatéraux conclus entre les Etats africains prévoient, parmi divers modes de règlement des différends, également la voie judiciaire. Ce sont soit des organismes arbitraux communs - tribunaux ou commissions créés par les Etats-Parties au traité - soit que les Parties se réportent aux normes juridiques universelles relatives à l'arbitrage international, ou à la Commission de Médiation, de Conciliation et d'Arbitrage de l'OUA. Certains traités prévoient un recours à d'autres centres internationaux d'arbitrage extra-africains. A titre d'exemple, on peut citer l'accord conclu entre la Société HOTAFRIC et la Cote-d'Ivoire (Abidjan, le 8 mars 1970) aux termes duquel tout différend qui aurait surgi entre les Parties sera soumis à la compétence du Centre International pour le Règlement des Différends relatifs aux Investissements de Washington. On soulignera que nombreux sont les Etats africains ayant signé la Convention pour le Règlement des Différends relatifs aux Investissements, entre Etats et Ressortissant d'autres Etats, du 18 mars 1965. ${ }^{17}$

Dans les traités, les modes judiciaires viennent généralement après les procédures diplomatiques. Rares sont ceux qui prévoient la soumission du différend à la Cour Internationale de Justice de La Haye, mais le plus souvent une réserve est formulée suivant laquelle le choix de la voie juridique n'est possible que dans le cas où les Parties ne sauraient régler le différend autrement, avec le concours d'autres institutions et procédures. Une telle solution est prévue, entr'autres, dans l'Accord portant création d'une commission de lutte contre le criquet en Afrique du Nord-Ouest et la Convention relative à la Navigation et à la Coopération Economique entre les Etats du Bassin du Niger. ${ }^{18} \mathrm{~A}$ cette occasion, on doit attirer l'attention sur les dispositions relatives au

17 La Convention internationale est entrée en vigueur le 14 octobre 1966. Conformément au Préambule de la Convention, le Centre de Washington doit fonctienner sous les auspices de la Banque Mondiale et dans son siège. Cf. la liste des Etats-membres du Centre International pour le Règlement des Différends relatifs aux Investissements. CIRDI /3/Rev.

18 Annuaire du Tiers Monde, 1976, p. 307; Droit International et ..., op. cit., p. 323. 
règlement des différends contenues dans l'Accord sur la lutte contre le criquet cité plus haut. L'article XVI dudit Accord statue qu'avant d'être porté devant la CIJ, le différend doit etre soumis à un comité composé d'un membre désigné par chacune des Parties au litige, et d'un président indépendant choisi par les membres du Comité. Les recommandations du Comité ne lient pas les Parties en cause mais celles-ci doivent reconsidérer la question qui est à l'origine du différend. Cette solution présente un intérêt incontestable parce qu'elle réunit des éléments de l'arbitrage et ceux de la conciliation. Bien que de par sa structure et son mode de constitution le Comité rappelle quelque peu un organe arbitral, son caractère reste pour autant consultatif. C'est une combinaison d'une structure assez formalisée avec une élasticité et liberté caractérisant ses résultats.

Il est caractéristique que la plupart des accords conclus entre les Etats africains déterminent en principe la compétence des organes judiciaires comme obligatoire, et ceci dans les termes suivants: "le différend sera porté, soumis", etc. Ainsi, l'art. 56 du traité instituant la Communauté Economique des Etats de l'Afrique de l'Ouest statue que dans l'impossibilité de régler le différend à l'amiable, ceclui-ci "sera porté devant le Tribunal de la Communauté". ${ }^{19}$ L'arbitrage prévu par l'Accord entre la HOTAFRIC et la Côted'Ivoire cité plus haut a un caractère nettement obligatoire. Il en est de même du Statut du Tribunal du Marché Commun Est-Africain et du Statut du Fonds Africain de Développement, les deux prévoyant l'obligation de recourir au règlement judiciaire pour les différends surgis entre le Fonds et les Etats-membres. Quant à la Charte de la Banque Africaine de Développement, elle prévoit également un recours obligatoire à l'arbitrage pour les différends où la Banque serait impliquée. ${ }^{20}$

La plupart des traités ou admettent l'institution ou instituent eux-mêmes des cours d'arbitrage permanentes, ou encore des commissions ou tribunaux arbitraux ad hoc créés conformément à une procédure semblable. Les procédures arbitrales se ressemblent également. Ces organes sont composés généralement de trois membres, un pour chaque Partie au litige et le troisième - président - est choisi conjointement. Ainsi, l'influence des Etats sur le jury est évidente. Parfois, les Parties au différend demandent aux organisations internationales ou même au Président de la Cour Internationale de Justice de désigner les arbitres.

Rares sont les accords où il est question de la base juridique du règlement. On constate, le plus souvent, que la juridiction est basée sur les normes universelles du droit international, les principes de justice et d'équité, ou encore sur d'autres normes désignées par les Parties au litige.

La revue des traités permet de se rendre compte que parmi les modes judiciaires de règlement des différends, le choix des Etats africains porte sur l'arbitrage; ces Etats sont également enclins à créer des tribunaux arbitraux. Parmi les rares exceptions, nous

19 Af rique Contemporaine, 1976, no 88, p. 20.

20 The Basic Documents ..., op. cit., p. 256 et suiv. 
pouvons citer l'institution du Tribunal du Marché Commun Est-Africain (organe judiciaire) et de la Cour d'Appel pour l'Afrique Orientale. ${ }^{21}$

Les modes de règlement des différends prévus par le droit international positif africain ne s'écartent pas, en principe des standards adoptés par le droit international universel, bien que certaines solutions proposées par les traités fassent penser à des modifications dans cette matière. Ce qui frappe ici c'est un recours fréquent des Etats africains Parties aux accords internationaux - à la procédure de règlement à l'amiable des différends. On y observe également le fait que les procédures judiciaires, et surtout l'arbitrage, sont citées fréquemment. Il est rare, par contre, que les Etats africains prévoient dans les traités un recours à la CIJ de La Haye, mais nombreux sont ceux qui stipulent que le différend serait soumis à la Commission de Médiation, de Conciliation et d'Arbitrage de l'OUA.

\section{Modes de règlement des différends - cas puisés dans la pratique des Etats africains}

L'étude et l'analyse de la pratique des Etats africains dans le processus de règlement des différends internationaux soulèvent des difficultés en raison de l'accès presque impossible et aux informations et documents. Ceci empêche surtout une analyse juridique du problème. Par contre, il est relativement plus facile d'accéder aux informations et documents concernant la pratique de l'Organisation de l'Unité Africaine en matière de règlement des différends; cette organisation qui réunit tous les Etats indépendants du continent devient une sorte d'indice et de résultante des tendances actuelles dans la pratique des Etats africains.

Il en résulte des documents et informations accessibles que l'OUA a contribué à la solution des 15 différends internationaux en recourant aux divers modes de règlement suivant le stade et l'étendue du litige. C'étaient des interventions effectuées par des organes supérieurs, mais il était fréquent que l'Organisation sollicitait la médiation de leaders africains. On citera le cas de la demande adressée au président du Soudan, M. Ismail Azhari, par la session de la Conférence des Chefs d'Etat et de Gouvernement qui s'est tenue à Kinshasa, en 1967. Ayant accepté cette demande, M. Azhari présidait la Commission Spéciale d'Enquête appelée à examiner et à déterminer les bases, les causes et la réalité matérielle sanctionnant les actions de "mercenaires blancs" au Congo et au Rwanda. ${ }^{22}$

Le fait d'évoquer divers modes de réglement des différends ne signifie pas que ces procédures ont été réellement utilisées, les Etats africains n'attachant pas une impor-

Cf. à titre d'exemple: Documentation française, 1970. Textes et Documents: Journal Officiel de la République Française, 12: II: 1978; L. B. Sohn, op. cit.; Revue Française de Droit Aćrien, 1963; Afrique Contemporaine, 1976, no 88, 1966, no 18; AFDI, 1971; Revue Juridique et Politique, 1965, no 2; Droit International ..., op. cit.; Annuaire de l'Afrique du Nord, 1976, p. 299. RGD, 1967, Chronologie des faits internationaux. 
tance excessive à la terminologie. Cet état de choses engendre parfois des complications, car à défaut de documents et vu l'accès difficile aux informations, il est impossible de déterminer les procédures utilisées en réalité. On notera, cependant que c'étaient toujours des procédures diplomatiques et jamais judiciaires.

Commençons par les négociations directes. C'est le mode de règlement des différends auquel les Parties peuvent toujours recourir bien que souvent la situation d'un différend le rend impossible. A plusieurs reprises, l'OUA appelait les Parties au différend à entamer les négociations directes, comme, p.ex., à l'occasion du conflit éthiopo-somalien, éthiopo-kenyan ou algéro-marocain. On citera le cas de la résolution relative au règlement pacifique d'un différend territorial entre l'Ethiopie et la Somalie. Le Conseil des Ministres de l'OUA a recommandé aux gouvernements - Parties au conflit - d'entamer des négociations en vue d'un règlement pacifique du différend. ${ }^{23}$ Il en était de même du litige opposant la Somalie et le Kenya où le Conseil des Ministres a appelé les Parties à "entamer des négociations le plus vite possible“. ${ }^{24}$ Dans les cas cités plus haut. L'ouverture des négociations était faisable grâce, par ailleurs, à l'armistice et le cessez-le-feu sollicités par l'OUA dans ses résolutions antérieures. Par contre, en ce qui concerne le différend entre le Ghana et la Haute-Volta, la Conférence des Chefs d'Etat et de Gouvernement a adopté une seule résolution où elle recommandait que les chef $s$ d'Etat des pays en litige "procèdent à des discussions directes en vue de trouver une solution du différend frontalier acceptable par les deux Parties".$^{25}$ La pratique de l'OUA tendait de plus en plus nettement à rehausser l'importance des négociations directes afin qu'elles deviennent le mode principal de règlement des différends. Ainsi, la $V^{\mathrm{e}}$ session extraordinaire du Conseil des Ministres de l'OUA qui s'est tenue à Lagos, en 1965, a adopté la résolution où toutes les Parties intéressées sont priées de régler et de résoudre tout malentendu fraternellement, par voie des négociations bilatérales. ${ }^{26}$ Il est vrai que la Charte de l'OUA cite les négociations parmi les modes de règlement des différends, cependant cette procédure n'est pas mentionnée dans le Protocole de Médiation, de Conciliation et d'Arbitrage. Néanmoins, la pratique a démontré son utilité incontestable, surtout qu'elle convient parfaitement à la tradition de "palabres africaines".

La pratique des Etats africains, membres de l'OUA, en matière de recours aux modes diplomatiques indirects de règlement des différends est très riche. L'OUA instituait souvent diverses commissions et missions auxquelles on attribuait la définition "de bons offices", "de médiation", "de conciliation" ou même "d'arbitrage".

La première commission que l'OUA qualifie comme "d'arbitrage" est celle instituée par les accords de Bamake relatifs au différend algéro-marocain. Sa tâche, aux termes desdits accords, devait consister à établir le responsable de l'ouverture des hostilités, à examiner le différend et, enfin, à proposer aus Parties les modes de son règlement. ${ }^{27} \mathrm{On}$ 
voit ainsi que le caractère de cette commission se rapproche nettement de la procédure d'enquête, et à celle de conciliation, par contre, sa ressemblance à une procédure d'arbitrage est plutôt faible.

On a également qualifié "d'arbitrage" une action du Conseil des Ministres de l'OUA en vue de régler un différend entre le Ghana et la République de Guinée (il était question de libération de diplomates guinéens retenus à aéroport d'Accra, qui devaient se rendre à une conférence de l'OUA), ainsi qu'une intervention du président zairois M. Mobutu dans un différend entre le Gabon et la Guinée Equatoriale. ${ }^{28}$ Toutefois, ni l'un ni l'autre cas n'offraient pas de possibilités d'une action arbitrale au sens strict de ce mot. Une telle nonchalance à utiliser la terminologie juridique est expliquée par les Africains comme une nécessité d'adopter une attitude pragmatique à l'égard de ce problème. Ainsi, p.ex., le président du Niger a suivi cette idée en déclarant à la session de l'OUA à Addis Ababa (1967) que "le fait que la commission soit de caractère de médiation ou de conciliation importe peu - il est essentiel, par contre, qu'elle puisse créer des possibilités permettant de mettre fin à la hostilité entre Etats ". ${ }^{29}$

Dans la plupart des différends où l'on note une ingérence de l'Organisation de l'Unité Africaine on a recouru à des procédures diplomatique indirectes, et la participation de l'élément tiers avait, le plus souvent, le caractère collectif (commissions). Ce n'est qu'à l'occasion de quatre différends que l'OUA s'en est remise à un médiateur. Ainsi, le rôle de médiateur dans le dífférend entre la Guinée et la Côte-d'Ivoire a été confié au président du Libéria, M. Tubman; la résolution en la matière adoptée par l'OUA stipule que cette dernière prie le président Tubman de bien vouloir "continuer la mission de conciliation qu'il a entreprise ". ${ }^{30}$ En réalité, le rôle de $\mathbf{M}$. Tubman était celui de médiateur-type assistant les Parties à trouver une telle solution politique du différend qu'elles pourraient accepter. On rappellera également un différend entre le $\mathrm{R}$ wanda et le Burundi où le rôle du président du Zaire, M. Mobutu, a été défini avec autant d'imprécision. A sa III ${ }^{\mathrm{e}}$ session extraordinaire, la Conférence de Chefs d'Etat et de Gouvernement a demandé au Parties au litige de "s'abstenir de toute action inamicale" et a prié le président Mobutu de "remplir une mission de bons of fices dans ce différend", sans préciser le caractère de l'action, d'autant plus que peu de temps après, une "mission de médiation" a été à son tour confiée au ministre des Affaires Etrangères zaïrois. ${ }^{31}$ Ensuite, le président Mobutu s'est vu confier à nouveau une autre "mission de médiation", cette fois couronnée de succès. Pour une fois, c'était un cas typique de médiation. Du 18 au 20 mars 1967, les Parties au différend, en présence du médiateur, se sont rencontrées à Goma où elles ont signé un accord mettant fin au conflit et instituant, entr'autre, une

27 Cf. Zbiór Dokumentów/Recueil de Documents/, 1963, no 9-10, pp. 1465-1467, op. cit.

28 Afrique Contemporaine, 1972, no 64.

29 OAU Secretariat. Resolutions and Declarations adopted by the Fourth Ordinary Session of AHG. Addis Ababa 1967.

30 Ibidem.

31 OAU Secretariat. Resolutions presented in the First Instance and accepted by the Third Session of AHG, Addis Ababa, 1966. 
commission de consultation permanente pour le règlement des différends qui surviendraient entre les deux Etats. ${ }^{32}$ On évoquera un cas de sui generis médiation où le Secrétaire Général de l'OUA a réussi de faire accepter par les Parties au différend tanzano-ougandais (dans sa phase de 1972) la médiation du ministre somalien des Affaires Etrangères laquelle a abouti à la signature d'un accord mettant fin au litige. Mais c'est par un échec que s'est terminée la mission de médiation confiée par l'OUA, en 1978, au ministre des Affaires Etrangères nigérien M. Joseph Garba et accomplie au cours de l'une des dernières étapes du différend entre la Somalie et l'Ethiopie relatif à ladite Corne d'Afrique.

Le fait que l'OUA ne confie que rarement les missions de médiation à une seule personnalité peut s'expliquer par les difficultés de faire accepter le candidat par les deux Parties au litige. Il est fréquent, par contre, que l'OUA institue des commissions ad hoc composées de plusieurs personnes comme c'était le cas du différend algéro-marocain. Au point de vue chronologique, c'était la première commission de ce genre instituté par la résolution adoptée en 1978 à la session extraordinaire du Conseil des Ministres de l'OUA à Addis Ababa, qualifiée injustement "d'arbitrage" ${ }^{34}$ Une autre commission ad hoc a été instituée à la III ${ }^{\mathrm{e}}$ session extraordinaire du Conseil des Ministres de l'Organisation de l'Unité Africaine qui s'est réunie du 5 au 10 septembre 1964 à Addis Ababa afin de régler le conflit congolais. ${ }^{35}$

En 1966, le Conseil des Ministres de l'OUA a institué une commission ad hoc appelée "Commission spéciale de bons offices" pour le règlement du diffèrend entre la Ghana et la République de Guinée, et composée de représentants du Congo, du Kenya et de Sierra Leone. Cette commission définie également comme "commission de médiation" ne peut en aucun cas être désignée comme "commission d'arbitrage. ${ }^{36}$ La tâche de la commission consistait à faire libérer les diplomates ghanéens, étudier la cause du litige, et à établir la situation réelle de citoyens ghanéens retenus ou séjournant en Guinée.

En 1971, le Conseil des Ministres de l'OUA a institué une Commission de médiation qui devait offrir ses services aux Parties au litige entre le Sénégal et la République de Guinée. Cette conception a été approuvée par la Conférence des Chefs d'Etat et de Gouvernement réunis à la VIII ${ }^{\mathbf{e}}$ session ordinaire où l'on a institué une Commission spéciale pour le règlement pacifique dudit différend, et composée de représentants des Etats suivants: Ethiopie, Algérie, Cameroun, Libéria, Mali, Mauritanie, Nigéria, Guinée et Sénégal. ${ }^{37}$ Les efforts de la Commission ont abouti à la signature de l'accord, au cours de la conférence de Mogadiscio, en mai 1972, mettant fin au différend. ${ }^{38}$

32 Le Monde, 21-22 III 1967.

33 Le Figaro, 8 II 1978.

$34 \mathrm{ECM} /$ Res. 1/I/, Organization of African Unity. Basic Documents and Resolutions.

35 Documentation Française, 1964, Articles et Documents, no 61586.

36 OAU. Council of Ministers. Resolutions of Ordinary and Extraordinary Session, Addis Ababa, 1965.

37 OAU Secretariat, Addis Ababa, 1971.

38 Le Monde, 31 V 1972. 
Pareillement, en ce qui concerne le litige opposant le Mali et la Haute-Volta, l'OUA a institué une Commission de médiation composée, à côté des Parties en litige, des représentants du Niger et du Togo. Sa tâche devait consister à 1) garantir la sécurité des citoyens d'un Etat se trouvant sur le territoire de l'autre, 2) obtenir l'évacuation des troupes armées de la zone faisant objet du litige, 3) trouver une solution du différend sur la base des documents juridiques relatifs à ce problème. Ensuite, la Commission a créé trois sous-commissions, à savoir a) à la Croix-Rouge, b) militaire, c) juridique. ${ }^{39} \mathrm{~A}$ en juger aux tâches que l'on vient de mentionner, la commission avait le caractère mixte comportant aussi bien des éléments de médiation que ceux de conciliation, de même que certains éléments propres à une commission surveillant l'exécution de l'armistice. La Commission s'est réunie le 18 décembre 1974, et son premier succès était la conclusion d'un "accord fraternel" mettant fin à une guerre de propagande. La réunion suivante de la commission a eu lieu le 18 juin 1975 à Lomé, en présence des présidents respectifs du Togo, du Sénégal, du Niger, du premier-ministre de la Guinée, du Secrétaire général l'OUA, et d'un représentant du président de l'Algérie; les Parties au litige se sont engagées à mettre fin au conflit et à régler celui-ci conformément aux recommandations de la Commission. En juillet de la même année, les deux Parties ont signé un accord relatif à "la paix permanente " ${ }^{40}$ bien que le problème de frontières - objet du litige n'ait pas encore été résolu.

En 1976, l'Organisation de l'Unité Africaine a encore constitué une commission de médiation avec la participation de l'Ouganda et de la Mauritanie, en vue du règlement d'un différend entre la Libye et la Tunisie.

L'un des organismes ad hoc créés récemment était le Comité chargé du règlement d'un différend entre l'Ethiopie et le Soudan, constitué à la XIV ${ }^{\mathbf{e}}$ session de la Conférence des Chefs d'Etat et de Gouvernement réunie à Libreville, en 1977. Aux travaux du Comité ont été invités les Etats suivants: Zambie, Sierra Leone, Togo, Algérie, Sénégal, Zaire, Nigéria, Cameroun et Gabon; lesdits Etats devaient oeuvrer en vue de "rechercher les voies et les moyens les meilleurs de régler ce différend, dans l'intérêt des Etats frères concernés en particulier, et de l'Afrique en général". Le Comité était tenu de présenter un rapport sur ses activités à la Conférence des Chefs d'Etat et de Gouvernement. ${ }^{41} \mathrm{~A}$ la même session de ladite Conférence, un autre comité ad hoc a été institué en vue du règlement d'un différend entre le Tchad et la Libye, composé des pays suivants: Sénégal, Algérie, Cameroun, Nigéria, Mozambique et Gabon. Conformément à la résolution respective, la Comité est obligé d'entreprendre "toutes les actions nécessaires, de rechercher toutes les voies et tous les moyens susceptibles de mener à bien la solution de ce grave problème " ${ }^{42}$ Dans les deux cas, le caractère du Comité n'a pas été défini, il semble, pourtant, qu'il était proche de la procédure d'enquête et de conciliation.

39 Le Soleil, 7 I 1975.

40 Afrique Contemporaine, 1975, no 77, no 81.

41 Documentation Française, 1977, Documents d'Actualité Internationale, no 49, p. 950.

42 Documentation Française, 1977, Documents d'Actualité Internationale, no 49, p. 950. 
Le caractère des procédures utilisées par l'OUA est souvent difficile à définir vu, entre autres, l'absence de documentation et d'informations. Dans la plupart des études, les auteurs s'en remettent à des informations fournies par la presse, d'où une certaine liberté à utiliser la terminologie, liberté qui souvent induit en erreur même les auteurs d'études. ${ }^{43}$

On pourra dire, toutefois, que dans le processus de règlement des différends, l'Organisation de l'Unité Africaine utilisait exclusivement les procédures politique et diplomatiques, qu'elle créait de nombreuses commissions ad hoc, en évitant des procédures formalisées. ${ }^{44}$ Borella estime que la formalisation des procédures utilisées par l'OUA aurait complètement paralysé son fonctionnement en matière de règlement des différends, bien qu'à une étape ultérieure, la formation des normes du droit international africain nécessiterait une définition plus formelle desdites procédures. Seul le processus de cette évolution fournit une chance réelle à l'établissement des relations pertinentes et cohérentes entre les normes du droit positif et la pratique des Etats africains. De toute façon, les méthodes utilisées par l'Organisation n'entrent pas dans la typologie classique des modes de règlement des diffèrends..$^{45}$ Plus précisément, il paraît que ces méthodes entrent quand même dans ladite typologie, bien que de par leur caractère, elles s'écartent quelque peu des modèles classiques.

Borelle est d'avis que le fonctionnement de l'OUA dans le processus de règlement des différends a trois caractéristiques principales: 1) presque tous les différends résolus l'ont été grâce à une autorité politique de leaders africains, même si une comission composée de plusieurs personnes a participé à leur règlement; 2) toutes ces procédures ont le caractère ad hoc; 3 ) lesdites procédures avaient toujours un caractère politique - c'étaient le plus souvent les négociations soit directes soit indirectes, avec la participation des Chefs d'Etats et de Gouvernement africains.

Ce rôle particulier des dirigeants africains est généralement souligné, ce qui reste incontestablement en rapport avec le caractère personnel d'un bon nombre des différends et, ce qui s'ensuit, avec le caractère personnel de leur règlement. On souligne généralement l'importance d'une intervention ad hoc de l'OUA qui serait impossible si les procédures étaient formalisées. Des preuves à l'appui en la matière ont été fournies par la Commission de Médiation, de Conciliation et d'Arbitrage. ${ }^{46}$

Il n'y pas de doute que la conscience d'appartenier à la "grande famille africaine", et un intérêt authentique porté au différend par le continent entier influe favorablement sur le processus de règlement pacifique des différends. La preuve en est, entre autres, un grand

43 T. Bensalah, L'Enquête internationale dans le règlement des conflits. Paris 1976, p. 134; J. P. Cot, La Conciliation internationale, Paris 1968, p. 267 et suiv.

44 Colloque de Bordeaux, Régionalisme et Universalisme dans le droit international contemporain, Paris 1977, p. 38.

45 F. Borella, le Système juridique de L'OUA, AFDI, 1971, p. 247 et suiv.

46 En réalité, aucun différend n'a été soumis à la Commission. Lo Protocole est resté lettre morte du droit. A la XIVe session de la Conférence des Chef s d'Etat et de Gouvernement, le fonctionnement de la Commission a été suspendu. 
nombre d'offres de médiation émanant de plusieurs pays africains. Bedjaoui estime que le fait de régler les différends dans le cadre de la "grande famille africaine" est une sorte de remède au caractère artificiel de la distinction des différends af ricains en politiques et juridiques. Le différend est considéré par les Etats africains comme un ensemble très complexe où s'entremêlent la matière politique et la matière juridique. Une telle attitude permet de se concentrer sur les modes effectifs de son règlement, sans se préoccuper du caractère de ceux-ci. ${ }^{47} \mathrm{La}$ nésessité de se plier à des principes formels et rigoureux entourant la plupart des procédures paralyse l'initiative des Etats africains. Il est donc naturel que ces derniers choisissent les modes qui conviennent mieux à la tradition de leur culture politique, celleci se trouvant à l'origine d'une nouvelle diplomatie africaine, ${ }^{48}$ dont la principale caractéristique est le retour à la "palabre africaine" dans le cadre de la "grande famille africaine". Morjane estime que c'est à cette dernière procédure que revient la première place parmi toutes les autres, dont témoignerait une évolution de l'attitude de l'Organisation de l'Unité Africaine à l'égard des modes de règlement des différends. ${ }^{49}$

La revue des procédures utilisées par l'OUA dans le processus de règlement pacifique des différends internationaux en Afrique permet de se faire une idée générale du problème de règlement pacifique des litiges par les Etats africains.

Les procédures appliquées aux différends où l'OUA n'à pas intervenu sont les mêmes que celles mentionnées plus haut lors d'une présentation du rôle de l'Organisation dans le processus de règlement des différends. Ceci concerne également les procédures utilisées à régler les litiges qui - bien que se trouvant dans le champ d'intéret de l'OUA -, étaient réglés sans son intervention. Ainsi, la préférence des Parties va aux négociations bilatérales - on citera, à titre d'exemple, les différends entre l'Algérie et la Tunisie, la République du Congo et la République Populaire du Congo, le Cameroun et la République Populaire du Congo, le Zaire et le Soudan, la Côte d'Ivoire et la Guinée, l'Egypte et la Tunisie, l'Egypte et le Zaire, l'Ethiopie et la Tunisie, le Ghana et le Togo, le Kenya et la Tanzanie, l'Ouganda et la Tanzanie, le Soudan et l'Egypte, enfin entre le Tchad et le Soudan. Nombreux sont les diffèrends où le rôle de médiateur est confié à d'éminents hommes d'Etat africains connus également en dehors de l'arène politique africaine, dont l'empereur Hailé Selassié, MM. Modibo Keita, Sékou Touré, Mobutu, Kaunda, Ngouabi, Tubman, et d'autres. Ensuite, on utilisait fréquemment les modes diplomatiques collégiaux, sous forme des commissions composées de plusieurs hommes d'Etat africains. Leur intervention relève soit de la technique de bons offices soit de celle de médiation, parfois même, elle glisse vers la procédure de conciliation.

Il arrive qu'une offre de médiation émane d'autres organisations internationales. Citons,

47 M. Bedjaoui, Le Règlement politique des différends africains, AFDI 1972, p. 94 et suiv.

$48 \mathrm{Ph}$. Chapal, Le Rôle de l'OUA dans le règlement des litiges entre Etats af ricains, Revue Algérienne des Sciences Juridiques, Economiques et Politiques, 1971, vol. VIII, no 4, p. 909.

49 K. Morjane, L'Organisation de l'Unité Africaine et le règlement pacifique des differends interafricains, La Haye, 1975, p. 54 et suiv. 
à titre d'exemple, un différend opposant la République Populaire du Congo et le Gabon qui a été réglé grâce à une conférence de la "table ronde" ayant réuni les chefs d'Etats et de Gouvernement des Etats-membres de l'Union Douanière de l'Afrique Equatoriale. Des cas pareils, pourtant, sont une exception et ont eu lieu, principalement, avant la création de l'Organisation de l'Unité Africaine. Certains différends entre les Etats arabes d'Afrique ont été résolus grâce à une intervention de la Ligue Arabe, tel le différend entre l'Egypte et la Maroc réglé au cours d'une session de la Ligue, tenue au Caire, en 1964. Un autre exemple de la participation d'une organisation sous-régionale au processus de règlement des différends en Afrique est une intervention réussie des Etats-membres du Conseil de l'Entente dans le différend opposant le Niger et le Dahomey, relatif à l'île Lété. Ajoutons que nombreux sont les litiges où l'on a eu recours aux bons offices d'un leader ou d'un chef d'Etat jouissant d'une grande autorité dans une sous-région concernée. Ceci explique une participation fréquente du président Mobutu au processus de règlement des différends en Afrique Centrale, des présidents Kaunda et Kenyatta en Afrique Orientale, ou, enfin, celle du président Tubman en Afrique Occidentale.

Pour des raisons citées plus haut, il nous serait difficile de présenter tous les éléments du processus de règlement pacifique des différends en Afrique. Ce processus se déroule de façon variée en fonction des différends où diverses procédures s'entremêlent dans un ordre changeant. Ce sont des procédures politiques ayant empêché les litiges de sortir du cadre africain. Depuis la création l'OUA, le cadre institutionnel de ce processus est, en principe, l'Organisation elle-même donnant la préférence aux procédures collégiales tandis que dans le cas des différends résolus sans son intervention, on observe une nette prédominance d'offices de médiation confiés aux leaders et aux hommes politiques africains.

La plupart des litiges entre les Etats af ricains ont été soit apaisés, immobilisés ou même gélés, soit résolus. ${ }^{50}$ Des 53 différends dont on possède une documentation ou information relativement complètes, 29 ont abouti à un accord, 16 ont été immobilisés ou apaisés, 6 n'ont pas été résolus et 2 différends se sont éteints avant qu'une action diplomatique ait éte entreprise. Ainsi, l'indice d'efficacité de la diplomatie africaine est plutôt élevé - dans différentes périodes, $85 \%$ des litiges ont été éliminés et par là-même ont cessé d'empêcher le développement des rapports pacifiques entre les Etats. Les Etats africains qui sont à l'étape de la recherche des modes de règlement des différends les plus efficaces ne dépassent pas, en principe, le répertoire de procédures connues en droit international classique, en lui rajoutant, souvent, un sens nouveau. Celui-ci reste en rapport étroit avec la nécessité d'élaborer de telles solutions qui, tout en étant basées sur la tradition, seraient en même temps conformes aux transformations s'opérant en Afrique. Ces tendances s'expriment, entre autres, par un recours fréquent des pays africains

50 On considère un différend comme résolu lorsque les Parties se sont mis d'accord et reconnaissent que l'affaire est close. Le différend immobilis est un différend qui engendre une sorte d'entente entre les Parties, le plus souvent muette, mais parfois aussi formelle, suivant laquelle lesdites Parties ne le considèrent par comme objet d'activités diplomatiques permanentes. 
à un règlement amiable des différends. Le règlement amiable des différends basé sur un compromis extra-juridique où l'influence exercée par les Parties sur le processus même est assez importante, s'est largement réflété aussi bien dans la pratique en matière de traités qu' aux activités diplomatiques des Etats africains. Ainsi, le règlement ex aeque et bono est mentionné, p.ex., dans le Protocole de Médiation, de Conciliation et d'Arbitrage. La Convention relative à l'Aménagement général du Bassin du fleuve Sénégal prévoit le règlement amiable des différends concernant l'interprétation et l'application de la Convention. Il en est de même du Traité instituant la Communauté Economique des Etats de l'Afrique de l'Ouest qui prévoit la procédure du règlement amiable des différends, tout comme la Convention relative à la Navigation et à la Coopération économique entre les Etats du bassin du Niger.

La pratique en matière des traités connaît un grand nombre de dispositions pareilles. Il en résulte de plusieurs d'entre elles que les Etats africains interprètent la notion de règlement à l'amiable des différends assez spécialement, s'écartant de la façon standardisée de comprendre le sens de ladite notion adoptée par le droit international universel. La pratique des Etats africains prouve que ceux-ci comprennent cette notion au sens le plus large, l'étendant également au négociations directes, à une entente directe, à un règlement amical, etc. Les Etats africains empruntent à la procédure amiable tout ce qui convient le mieux à leurs traditions de culture, enfin à la personnalité africaine, et notamment, l'absence de formalisme juridique, ainsi que le recours aux notions de fraternité, communauté et équité. Il paraît donc que ce sont les principes remontant à un passé historique commun, à la tradition, culture, religion et, enfin, à la communauté d'intérêts qui font que les Etats africains persévèrent dans leur tendance à choisir un règlement amiable des différends estimant que ce dernier signifie également sinon avant tout la conclusion d'un accord bilatéral et direct entre les pays intéressés.

La revue de problèmes concernant le règlement des différends internationaux en Afrique (cas puisés dans la pratique relative aux traités, et exemples d'actions entreprises par des Etats en cette matière) permet de formuler une opinion sur la formation de la coutume régionale africaine concernant le règlement des différends internationaux entre les Etats de ce continent. Ces actions sont relatives non seulement à une modification concernant la matière du droit international, mais également à la sphère de ses formes et structures. La pratique ci-dessus tendant à une déformalisation optimale des procédures connues du droit international universel, ce n'est donc pas par hasard que parmi les procédures de règlement des différends citées dans la Charte de l'OUA et le Protocole de Médiation de Conciliation et d'Arbitrage il n'est guère question de voie judiciaire bien que les accords internationaux conclus antérieurement par les Etats africains mentionnent les modes judiciaires (il s'agissait, surtout, de l'arbitrage, rarement de la juridiction au sens étroit). Cette pratique est confirmée par le fait que jusqu'à l'heure actuelle, il n'y a qu'un seul différend entre les Etats africains (Libye contre Tunisie) qui a été soumis a la compé- 
tence de la Cour Internationale de Justice. La tendance à une déformalisation se rapporte non seulement aux formes et structures des mécanismes de règlement des différends, mais également à la matière juridique. Engagés au règlement des différends, les Parties se montrent mal disposées quand il s'agit de s'en référer au droit international, même si l'existence d'un droit subjectif en matière du différend ne soulève pas de doutes. Dans la pratique du réglement de litiges, les Etats africains adoptent souvent le principe ex aeque et bono - principe relatif au règlement à l'amiable compris au sens large auquel ils donnent une interprétation particulière. On rappellera que le comité chargé du règlement des différends, constitué en 1977 à une session de la Conférence des Chefs d'Etat et de gouvernmement de l'OUA avait le caractère tout à fait non formalisé, et son unique principe directeur est une directive pragmatique lui adjoignant de trouver le mode le plus efficace de règlement du différend.

Dans le processus de règlement des litiges, la pratique des Etats africains se distingue par une préférence accordée aux diverses commissions dont le caractère est difficile à définir du point de vue des procédures connues du droit international universel. Ce sont généralement des commissions réunissant des leaders africains et des autorités politiques du continent dont les activités restent en rapport avec la tradition de la "palabre africaine" et des "pélerins africains de la paix". C'est une sorte de "tribunal de sages africains" oeuvrant sur la base des principes d'équité, de justice et des liens qui émanent du sentiment de communauté. L'efficacité de ces modes est non négligeable, étant donné un degré particulier de la personnalisation des différends en Afrique. La coutume qui est en train de se fermer correspond donc aux réalités internationales du continent. Ceci est valable également lorsqu'il est question d'empêcher les différends de dépasser le cadre africain, et de les régler dans ce cadre. Une telle pratique a trouvé la confirmation dans un bon nombre de résolutions adoptées par tous les Etats africains-membres de l'OUA. ${ }^{51}$ Un modèle du système africain de règlement des différends qui est en train de se fermer est actuellement au stade de la creation de qualités nouvelles dans les relations internationales en Afrique. Elles se caractérisent, entr'autres, par le fait que la plupart des différends dégnèrent en des processus de controverse qui trainent en longueur et ont une intensité variable, mais qui portent, non obstant, un préjudice au maintien de la paix et de la sécurité internationales. Voilà pourquoi on recherche des solutions, principes, règles et instruments qui exclueraient l'anarchie et l'insubordination du domaine des relations internationales.

51 Mp.Rez./AHG/16/I, Res.ECM/9/V. 
firstly, the economic reforms are in part merely the pragmatic lessons learned from past failures; secondly, most of the economic reforms only serve as temporary models; thirdly, the Chinese economic reforms remain integrated into a basically socialist system; fourthly, other communist countries have already established and carried out similar reforms and, fifthly, the reforms find their theoretical background in Lenin's theory of state-capitalism.

\section{International Disputes in Africa}

\section{By Maria Magdalena Kenig}

The paper discusses problems of the peaceful settlement of international disputes in Africa.

About sixty disputes are discussed which arose in the years 1945-1985. This study attempts to establish the rôle and contribution of African states to maintenance of security in Africa, as well as to define the nature of the process from the point of view of the creation of norms of regional law in Africa. One of the hypotheses put forward states that there is a clear relationship between the processes for the settlement of international disputes in Africa and the overall development of contemporary international relations. Studies of importance for a discussion of the settlement of international disputes in Africa have been attempted at two levels, i. e. at the level of relevant African positive international law and at the level of the practical measures taken by states.

The survey of problems of the settlement of international disputes in Africa permits the conclusions that African states have created institutions, principles and norms of a regional legal order. This activity comprises not only modifications relating to substantive international law but also to its forms and structures. The custom developed in this field is accepted to the African community.

As a rule, African states have adopted diplomatic procedures, whose characteristics and nature are difficult to define. They do, however, possibilities of achieving far-reaching compromises and political arrangements on controversial issues, which clearly contributes to keeping these processes of settlement within a strictly African framework.

It is not easy to know the legal nature of the ad-hoc procedures utilised by African states. Despite a reluctance to adopt formal rules, there are some tendencies to establish principles which govern this area of international relations. These principles are based on African tradition in which African diplomacy is rooted. 